\title{
Ethnologies
}

\section{Filmer les pratiques de soin dans la consultation médicale en acupuncture}

\section{Une approche ethno-cinématographique}

\section{Gilles Remillet}

Volume 33, numéro 2, 2011

URI : https://id.erudit.org/iderudit/1015027ar

DOI : https://doi.org/10.7202/1015027ar

Aller au sommaire du numéro

\section{Éditeur(s)}

Association Canadienne d'Ethnologie et de Folklore

ISSN

1481-5974 (imprimé)

1708-0401 (numérique)

Découvrir la revue

Citer cet article

Remillet, G. (2011). Filmer les pratiques de soin dans la consultation médicale en acupuncture : une approche ethno-cinématographique. Ethnologies, 33(2), 99-121. https://doi.org/10.7202/1015027ar
Résumé de l'article

Dans la consultation d'acupuncture, les aiguilles remplacent les médicaments du médecin allopathe. Ainsi, le traitement n'est pas différé, mais s'effectue plutôt sur-le-champ. Dans ces conditions, quelle est la place accordée au corps, à la parole du malade et à ses émotions dans la construction de " sa maladie » ? Quels en sont les impacts pour sa prise en charge thérapeutique ? Cette médecine propose-t-elle un autre modèle de mise en récit des maux et de la maladie ? Au-delà des résultats mêmes de l'étude, cet article tente d'évaluer l'impact du dispositif filmique comme mode de connaissance anthropologique ainsi que les possibilités heuristiques qu'offre le film pour une anthropologie des pratiques thérapeutiques en situation clinique. Nous essaierons ainsi d'aller plus loin dans ce domaine de l'anthropologie visuelle, encore peu exploré. 


\section{FILMER LES PRATIQUES DE SOIN DANS LA CONSULTATION MÉDICALE EN ACUPUNCTURE}

Une approche ethno-cinématographique

Gilles Remillet

Université Paris Ouest Nanterre La Défense

"Le film est plus une façon de placer les choses dans un contexte que de créer une histoire. Un renouveau de l'œil.»

Johan van der Keuken (1998: 29)

\section{Genèse de la recherche}

Une étude réalisée en France en 2002, portant sur l'incidence des représentations sociales de l'épilepsie dans la relation médecin/ malade en milieu hospitalier, concluait à l'existence d'une attitude médicale récurrente consistant à minimiser l'importance du vécu et des interprétations des malades : "La médecine tend à considérer tout ce qui touche l'homme dans sa globalité, son projet de vie, comme des propos philosophiques et spéculatifs hors de son champ d'intérêt. En s'opposant par principe au sens commun, en niant sa réalité, sa cohérence et son efficacité, la médecine perd l'occasion de potentialiser ses effets » (Aledo-Remillet 2004 : 237). C'était le sens même du récit de la maladie par le patient qui tendait à être refoulé dans la " catégorie du subjectif " plutôt que d'être pensé comme un possible matériel de travail thérapeutique pour le médecin.

Ce premier travail sur le statut accordé à la "parole profane " dans la relation médecin/malade en situation clinique a éveillé ma curiosité ethnologique et j'ai voulu savoir ce qu'il en était de ce récit subjectif du patient dans la relation médecin/malade en acupuncture, médecine qui tend à se présenter soit en réaction, soit en complément 
de la médecine scientifique biomédicale ${ }^{1}$, comme plus ouverte à la démarche de soin intégrant une compréhension plus globale de l'homme malade et où le champ du corps, des émotions et de la parole semble tenir une place primordiale. Et cela, plus précisément, dans une consultation médicale d'acupuncture où la prise en charge thérapeutique n'est pas différée mais s'effectue plutôt "sur-le-champ ", ici, les aiguilles de l'acupuncteur remplaçant les médicaments du médecin allopathe, quelle est la place accordée au corps, à la parole du malade et à ses émotions dans la construction de "sa maladie "? Comment le malade arrive-t-il à mettre en mots ses maux? Que ditil de lui et qu'est-il amené à dire à travers sa maladie ? Comment le médecin traduit-il ou retraduit-il ce récit individuel du malade face à sa maladie et qu'en fait-il ? Cette médecine, parfois qualifiée de « douce " (Benoit 1998), de "parallèle " (Schmitz 2006), d' " alternative " (Wunenburger 2006) ou encore de « non conventionnelle (Candelise 2008), propose-t-elle vraiment un autre modèle de mise en récit des maux et de la maladie?

Tel est l'objet de la présente réflexion, qui se situe dans le contexte d'une " ethnologie du proche " et de la société française contemporaine. C'est donc à partir de consultations observées auprès de médecins acupuncteurs français que je tenterai un retour réflexif sur l'objet d'étude. Dans cet article, j'exposerai comment j'ai choisi d'avoir recours au film comme pratique d'enquête ethnographique et j'évaluerai dans quelle mesure l'observation filmée des pratiques de soin permet de saisir quelques-unes des composantes essentielles de la relation thérapeutique en acupuncture.

1. La biomédecine «[...] renvoie à un cadre théorique explicatif de la santé et de la maladie issu des sciences biologiques. Les propriétés vitales (dont la pathologie fait partie puisque la maladie est une forme de la vie) sont ramenées à des propriétés physico-chimiques. Nous nous situons depuis le XIXe siècle au sein d'un même paradigme scientifique, dont le champ d'exploration s'est fantastiquement élargi avec la microbiologie, la biochimie, la biophysique, la biologie moléculaire, la génétique, l’immunologie, etc. » (Mino 2006 : 81). 


\section{La consultation d'acupuncture en milieu libéral et hospitalier comme terrain d'enquête : la place du " tiers ethnologique "}

\section{Le choix des médecins et de leurs patients}

Le choix des médecins acupuncteurs étudiés et de leurs patients s'est essentiellement porté sur ceux que je côtoie par l'intermédiaire du réseau professionnel de mon épouse (elle-même médecinacupunteur), tous membres de l'Association française pour l'étude et la recherche en acupuncture (AFERA). Créée en 1973. son siège se situe à Nîmes (Gard) et rassemble plus de 100 médecins répartis dans toute la France. En effet, c'est d'abord en tant qu' époux " que j'ai régulièrement assisté depuis 15 ans aux congrès annuels, repas, colloques et réunions diverses organisés par cette association. Lors de ces nombreuses occasions, j'ai donc pu créer des liens durables de sympathie avec certains médecins et mon statut de chercheur ethnologue-cinéaste, mon intérêt pour le champ de la médecine, de la maladie, comme mes recherches ethnologiques, ont souvent fait l'objet de discussions fournies.

Leur intérêt pour les sciences sociales ne s'est d'ailleurs pas démenti lorsque l'association a décidé de commander en 2001 une étude sociologique sur la pratique de l'acupuncture en France destinée à faire valoir auprès des services publics en charge de la santé une meilleure visibilité institutionnelle de cet exercice médical particulier (Desjeux 2001)². Ainsi, lorsque j'ai fait part aux médecins les plus proches de ma volonté de faire cette étude sur la relation médecin/ malade en acupuncture, je n'ai trouvé que des échos favorables et l'attente, selon leurs termes, d'un "regard critique et distancié sur leur travail». Fort de cette acceptation et sur les conseils de mes premiers informateurs médecins, j'ai étendu ma requête à l'ensemble des membres de l'association en diffusant par courriel, grâce à l'aide de la secrétaire de l'AFERA, le projet d'enquête rédigé sous la forme d'une page synthétique présentant la problématique et surtout les principes méthodologiques de l'enquête qualitative ethnologique. J'insistais notamment sur le fait que cette étude nécessitait une présence suivie sur le long terme dans les cabinets médicaux et que

2. Sur les questions que posent l'institutionnalisation de l'acupuncture dans le système médical en France, le lecteur pourra également se reporter à la thèse de Ronald Guilloux (2006). 
le recours à l'observation participante sous la forme d'observations filmiques et/ou directes des consultations impliquait de fait l'accord préalable des médecins comme des malades. Enfin, je signalais également l'intérêt majeur pour la recherche de pouvoir mener des entretiens (parfois filmés) auprès des médecins et de leurs patients à partir des images tournées lors des consultations.

Cet appel lancé au plus grand nombre des médecins n'a pas finalement trouvé l'écho escompté ; parmi les réponses négatives reçues, les motifs portaient tout aussi bien sur la charge de travail supplémentaire que pouvait représenter un ethnologue pendant les consultations, que sur la délicate question soulevée par le respect du secret médical, les médecins ne voulant pas s'exposer ou exposer leurs patients au regard d'un " tiers ethnologique ", présence jugée susceptible de transgresser le code de déontologie médicale hippocratique. En fait, les médecins réfractaires mettaient à jour les difficultés majeures rencontrées par bon nombre d'ethnologues enquêtant sur les pratiques de soin en situation clinique, tant dans le milieu hospitalier (Vega 2000 ; Pouchelle 2003 ; Fainzang 2006) que dans le cadre de consultations médicales privées (Sarradon-Eck 2008), et auxquelles je me confrontais à mon tour. En quête d'autorisations légales, j'ai donc déposé une demande au Conseil de l'Ordre des médecins de ma région afin d'obtenir de cette instance officielle une légitimité institutionnelle conforme aux attentes du milieu enquêté. Après quelques semaines d'attente, ma demande d'autorisation a été rejetée au motif principal que «la présence d'un tiers au cours de la consultation est une immixtion dans la relation médecin/malade ».

Craignant que cette réponse ne remette en question ma recherche, j'ai pris contact avec un médecin généraliste, lui-même filmé lors de ses consultations par une cinéaste documentariste en $2008^{3}$. Après discussion, ce dernier m'a conseillé de faire signer des autorisations de droits à l'image avant le tournage et après le montage des films auprès des patients et des médecins. Après investigations, j'ai pu me rendre compte que le Conseil de l'Ordre n'avait qu'un avis consultatif à donner, et qu'en fait les soucis majeurs auxquels je risquais de me confronter pourraient bien être plus liés à des plaintes de médecins qu'à celles de patients mécontents. J'en ai donc informé les médecins-

3. Il s'agit du film d'Hélène de Crécy, La consultation, 90 min, 2006, Producteur Arturo Mio. 
acupuncteurs initialement favorables au projet et, après concertation, nous avons convenu de poursuivre cette recherche toujours dans le plus grand respect des patients en adoptant cet usage des signatures d'autorisation de tournage afin de protéger les protagonistes de l'étude - patients, médecins et ethnologue-cinéaste - d'une éventuelle plainte au Conseil de l'Ordre des médecins.

Finalement, l'enquête qualitative de terrain s'est déroulée de mi-septembre 2009 à juin 2010 et a porté sur six médecinsacupuncteurs, quatre hommes et deux femmes, exerçant en milieu libéral. Tous pratiquent dans la région Languedoc-Roussillon, dans les départements du Gard (Nîmes), de l'Hérault (Montpellier) et de l'Aude (Perpignan). L'un des médecins, bien qu'exerçant aussi en libéral, a préféré m'ouvrir les portes de sa pratique de l'acupuncture en milieu hospitalier (CHU), contexte plus propice selon lui à l'acceptation d'un tiers dans la relation médecin/malade. En effet, cette consultation étant aussi le lieu de formation des apprentis médecins-acupuncteurs, les patients étaient déjà plus habitués à la présence d'un tiers et la mienne paraissait moins incongrue.

Afin de ne pas entraver les médecins dans l'organisation de leur travail quotidien, nous avons convenu que mes périodes d'observation dans les cabinets libéraux se limiteraient à une journée par semaine et à une demi-journée en milieu hospitalier. Bien que restrictive sur le plan de l'insertion dans le milieu à étudier, cette contrainte m'a permis de suivre le traitement des patients sur le long terme, de plusieurs semaines à plusieurs mois. Les rendez-vous étaient pris en concertation avec l'ethnologue toujours sur les mêmes créneaux journaliers, ce qui permettait d'instaurer une relation de confiance toujours plus grande au fur et à mesure qu'avançait le traitement ; cela est allé jusqu'à me permettre de revoir la plupart des patients chez eux pour des entretiens dont certains ont également été filmés.

Le choix des patients enquêtés ne s'est pas porté a priori sur des pathologies particulières ni sur une catégorie socioculturelle type. Si certains médecins ont préféré ne pas sélectionner les patients en amont ( [...] on fera avec le tout-venant »), d'autres ont en revanche souhaité faire le tri parmi leurs patients, ne sollicitant que ceux susceptibles d'être sensibles à l'expérimentation ethnologique. Dans le premier cas de figure, cela me permettait d'assister à ce que les acupuncteurs 
désignent dans leur milieu professionnel par des " premières fois "; il s'agit en fait de consultations de patients qu'ils ne connaissent pas ou qui n’ont jamais été soignés par acupuncture. Je reviendrai sur l'importance pour l'enquêteur d'assister à ces premières consultations, primordiales pour la question de la construction du lien thérapeutique et du récit de la maladie. Dans le second cas de figure, ce recrutement sélectif des patients en amont m'a permis d'entrer en contact avec une patientèle d'habitués ayant instauré avec leur médecin une relation de soins datant parfois de plus de 30 ans, ce qui, d'un point de vue méthodologique, permettait l'analyse comparative avec la catégorie des "premières fois ». Enfin, grâce aux consultations observées en milieu hospitalier, pleinement remboursées par la Sécurité sociale contrairement à leur mauvaise prise en charge en médecine de ville, j'ai également pu avoir accès à une population de patients moins favorisés, ne fréquentant que très rarement les cabinets d'acupuncture de médecine libérale.

Aussi, le nombre des consultations étudiées a été fixé non pas dans une optique statistique mais plutôt dans un souci qualitatif fondé sur le principe épistémologique de la saturation de l'information relatif à chaque méthode utilisée : observations filmiques, observations directes et entretiens. Ainsi, les patients enquêtés sont au nombre de 113 , ce qui représente 135 consultations, 108 en milieu libéral et 27 en milieu hospitalier, dont 75 ont fait l'objet d'un enregistrement vidéographique constituant un corpus filmique d'une centaine d'heures de rushes, tandis que les 60 autres consultations ont été observées directement et transcrites sous la forme de notes de terrain. Enfin, plus de 30 entretiens systématiquement enregistrés au magnétophone ont été menés auprès des patients et des médecins afin de confronter le niveau du discours, des représentations de la maladie, à celui des pratiques de soin. 


\section{Ethnographie filmée des pratiques de soin : le corps, le cadre et la parole}

En tant qu'ethnologue-cinéaste, j'ai accordé une place centrale au film comme outil d'investigation ethnographique pour la description, l'analyse et la restitution des récits de la maladie et des pratiques thérapeutiques observées entre les médecins et les malades. L'outil audiovisuel m'a semblé en effet tout à fait approprié à cet objet d'étude dans la mesure où l'investigation vidéographique permet de saisir la complexité des interactions sociales (paroles, gestes, attitudes, postures, façons de dire et de faire, mises en scène de soi) observées durant les consultations médicales comme lors des entretiens filmiques.

J'aimerais maintenant revenir sur la manière dont se sont élaborés les premiers tournages à partir desquels j'ai pu expérimenter le choix du dispositif filmique le plus approprié pour filmer les consultations et certains entretiens. Je prendrai pour exemple la façon dont s'est élaboré le film Les aiguilles au fil des maux (2010), réalisé en collaboration avec un patient venu consulter un acupuncteur de l'Hérault un an auparavant.

J'ai donc commencé par faire un premier entretien semi-directif de ce patient chez lui (d'une durée d'environ 2 heures) dans l'intention de produire des données discursives sur le vécu de sa maladie et sur son rapport au milieu médical. Pour ce faire, j'ai mis au point un guide d'entretien assez souple, avec un certain nombre de grandes thématiques que je voulais aborder, notamment, le vécu de la maladie, l'itinéraire thérapeutique, le choix du médecin et du traitement par acupuncture, le rapport entre le malade et son entourage (familial, amical, professionnel), le rapport entre le malade et les médecins, le rapport au corps, le sens donné à la maladie, le rôle joué par le malade dans sa guérison, etc. J'ai donc privilégié une modalité d'entretien qui puisse se dérouler sur le ton de la conversation et non pas sur celui de l'interrogatoire, qui me semblait en fait trop proche de la pratique même du médecin observée lors de la consultation.

Pour filmer cet entretien, j'ai donc opté pour une caméra sur pied, pour le plan-séquence et pour le maintien d'un seul cadrage fixe en plan moyen délimitant le visage du malade et les mouvements de ses mains, face à la caméra durant tout l'entretien. Ce dispositif technique 
et ce choix de réalisation minimaliste devaient me permettre de n'avoir à intervenir sur la caméra qu'en cas de force majeure, pour ne pas rompre la relation d'intimité qui s'installait progressivement au fil de l'entretien. De plus, ce procédé me permettait de me situer à côté de la caméra, à visage découvert, face à mon interlocuteur, afin de maintenir avec lui une posture d'enquête empathique pour que la qualité de mon écoute et la liberté de ses paroles puissent pleinement s'exprimer. Cet entretien a donc eu lieu en début d'après-midi, à un moment de la journée que choisit généralement cet enquêté pour se reposer et donc, de lui-même, il a voulu apparaître filmé allongé sur son canapé, " comme chez le psy! »a-t-il précisé, de façon à être confortablement installé pour me parler. On remarquera ici l'analogie faite sur le ton de la plaisanterie entre la posture de "l'enquêteur écoutant » et celle d'un psychanalyste, le patient allant même jusqu'à confier à l'ethnologue à la fin de l'entretien le bien-être éprouvé par le patient d'avoir parlé de sa maladie. Puis, dans un deuxième temps, j'ai filmé ce même malade lors de sa cinquième consultation chez son médecin-acupuncteur qui exerce près de Montpellier en cabinet libéral.

La consultation en acupuncture se déroule selon une " automise en scène ${ }^{4}$ spatio-temporelle relativement stable. En effet, cette consultation qui dure environ une heure peut être divisée en plusieurs temps distincts que j'ai pu retrouver chez les autres acupuncteurs enquêtés avec des variantes relativement faibles liées à des structurations de l'espace de travail différentes (séparation ou non du lieu de paiement en fin de consultation, séparation ou non du lieu d'écoute du patient de celui de la puncture dans une autre pièce) :

- l'attente du patient et son accueil par le médecin ;

- le temps de "l'interrogatoire " qui est le moment où le patient expose sa plainte, ce dialogue qui s'engage entre le patient et son médecin se déroulant autour du bureau de ce dernier ;

4. Cette notion désigne en cinématographie documentaire «[...] les diverses manières dont le procès observé se présente de lui-même au cinéaste dans l'espace et dans le temps. Il s'agit d'une mise en scène propre, autonome, en vertu de laquelle les personnes filmées montrent de façon plus ou moins ostensible, ou dissimulent à autrui, leurs actes et les choses qui les entourent, au cours des activités corporelles, matérielles et rituelles. L'auto-mise en scène est inhérente à tout procès observé. Aussi ne doit-elle pas être confondue avec l'auto-soulignement, qui n'en est qu'une forme particulière » (France 1989 : 368). 
- puis vient le moment de l'examen clinique (examen de la langue, prise du pouls) ;

- la séance de puncture proprement dite qui implique un déplacement des protagonistes dans l'espace du cabinet puisque ces actions se déroulent sur la table d'examen après que le patient se soit déshabillé ;

- là, une fois les points d'acupuncture piqués sur le corps du patient, il est laissé seul avec les aiguilles une vingtaine de minutes avant d'être "dépiqué ";

- une fois le patient rhabillé, la consultation se poursuit de nouveau autour du bureau du médecin et se conclut par la prise d'un nouveau rendez-vous et par l'accompagnement du patient à la porte du cabinet.

Pour filmer ces différents temps de la consultation, j'ai choisi un dispositif scénique qui puisse rendre compte des interactions corporelles entre les agents et restituer l'intégralité des interactions verbales entre le médecin et son patient. En effet, l'objet principal de l'étude étant le récit de la maladie et la mise en mots des symptômes, il fallait que les différentes modalités d'usage de cette parole durant la consultation soient intégralement enregistrées du côté du médecin comme du côté du malade. J'ai donc choisi de filmer avec deux caméras selon le principe du champ/contre-champ de façon à pouvoir restituer dans la continuité sonore les diverses situations d'échanges entre les protagonistes. Cette solution m'a permis de synchroniser en plan-séquence deux caméras. Une première caméra sur un pied en plan fixe filme le médecin, soit en cadrage serré sur son visage, soit en cadrage élargi, le présentant dans son espace de travail assis à son bureau, le patient en face. À l'aide d'une deuxième caméra - à partir d'un «poste d'observation sédentaire » (Gueronnet 1987 : 13) - située à équidistance des protagonistes, je filmais, toujours en plan-séquence, et soulignais au moyen du zoom optique chez les deux protagonistes ce qui me semblait utile au cours des interactions observées. Cette stratégie filmique me permettait également de venir filmer près des corps et de souligner les gestes de l'examen clinique et de la puncture sans que le registre du champ sonore, relatif aux paroles accompagnant ces techniques, en pâtit. Ce dispositif présente l'avantage de faire varier les points de vue sur l'action sans que le cinéaste n'ait à se déplacer autour des agents pendant les temps de face-à-face (interrogatoire et fin de la consultation) où s'installe 
entre eux une relation d'écoute et d'intimité particulières, séquence thérapeutique durant laquelle tout déplacement du cinéaste-chercheur dans l'espace ritualisé du cabinet médical tend à être perçu comme illégitime et invasif, entraînant des comportements "profilmiques " (France 2006 : 373) allant du simple regard à la caméra, à l'arrêt brutal des discussions entre le médecin et le patient, délaissées pour observer les déplacements du cinéaste.

De plus, ce choix de réalisation, bien qu'a priori très strict et assez limité sur le plan de la mise en scène, permet de ne pas " prémonter " le film au tournage en lui donnant un sens déjà trop induit par la perception du cinéaste impliqué émotionnellement par la situation filmée. En revanche, cette posture méthodologique adoptée au moment du tournage implique finalement un temps plus long de post-production (montage, étalonnage et mixage du son), compte tenu des rushes issus des deux caméras à synchroniser. Toutefois, en analysant en parallèle les images de la consultation filmée in situ et le contenu de l'entretien filmique, j'ai été frappé de constater combien certains sujets étaient récurrents bien que différemment mis en scène. Dans un premier montage, j'ai restitué sur le plan filmique cette complémentarité épistémologique du discours et de la pratique. Le croisement des données produit un éclaircissement mutuellement des deux pratiques, lors de la confrontation au montage du film. On y perçoit le déroulement phénoménologique entre le vécu subjectif et la relation au médecin, à la maladie, au corps.

J'ai eu recours à ce même procédé filmique pour rendre compte de la plupart des consultations. Cependant, en milieu hospitalier, elles ne se déroulaient que rarement dans les mêmes pièces, les salles d'examens étant simultanément à disposition de trois autres médecins - acupuncteurs du service. Il m'était donc impossible d'installer au préalable mon dispositif filmique comme dans les cabinets médicaux de ville où, pour faciliter mon travail de préparation technique et ne pas retarder le rythme des rendezvous, une salle identique m'était attribuée le temps de ma présence journalière. Dans le cas des consultations hospitalières, j'ai opté pour une observation filmique "flottante" (Petonnet 1982 : 37), dans une optique exploratoire privilégiant la stratégie du plan-séquence, faisant correspondre le temps de mes enregistrements filmiques à celui de la consultation afin de ne pas rompre la continuité sonore 
des échanges verbaux entre les protagonistes : le médecin, le malade et l'apprenti acupuncteur en stage de formation. Dans cet espace, seuls quelques postes d'observation situés en périphérie du bureau et de la table d'examen m'étaient autorisés. Là aussi, les déplacements dans l'espace d'interaction triangulaire entre le médecin, son patient et l'apprenti acupuncteur étaient proscrits ; je profitais des déplacements des enquêtés du bureau vers la table d'examen pour changer de point de vue sur l'action, soulignant tour à tour les gestes du médecin sur le patient et ses commentaires adressés au futur acupuncteur. Ici, comme dans les cabinets de ville, les textures sonores des paroles, gestes, bruits du matériel médical étaient primordiales à restituer. $\mathrm{Ne}$ possédant pas d'ingénieur du son, afin de réduire l'équipe de tournage au minimum, j'ai eu recours à un micro HF omnidirectionnel placé sur le médecin, qui permettait d'avoir certes toujours la même valeur de plan sonore, le gros plan, mais que j'ai mixé avec une valeur de plan sonore plus large d'ambiance en répartissant le micro HF et celui de la caméra sur deux pistes distinctes. Ce procédé de tournage permettait ensuite au montage de mixer les pistes et de faire varier l'importance des valeurs de plans, de hiérarchiser l'information sonore en fonction de l'action, par exemple de donner la priorité à l'intelligibilité des paroles lors de l'interrogatoire ou à l'ambiance sonore des gestes pendant la séance de puncture. Enfin, l'utilisation du micro HF m'a aussi donné la possibilité d'enregistrer à distance les négociations de ma présence entre le médecin et les patients avant la consultation, comme les commentaires de ce dernier dans le couloir, lieu officieux, mais couramment utilisé, pour transmettre son savoir professionnel à son apprenti stagiaire.

\section{Montage(s) thérapeutique(s) : dire, écouter, interpréter}

\section{Point de vue de l'acupuncture sur la maladie}

L'acupuncture est une médecine d'origine chinoise dont les fondements théoriques et certaines techniques de soin n'ont cessé d'évoluer depuis 4000 ans. En retracer l'histoire déborde le cadre de cet article ; je me limiterai donc à en présenter ici les grands principes de manière synthétique. Cette pratique médicale n'a été connue que tardivement en Occident ; les premiers commentaires en ont été faits par les jésuites au XVIIe siècle et il faut attendre 1934 
et les traductions de Georges Soulié de Morant (consul de France en Chine) pour que cette médecine devienne autre chose qu'une simple curiosité exotique et puisse trouver une application concrète en France à partir des années $1960^{5}$.

Cette médecine est basée sur une conception énergétique du corps qui fait appel à deux grands principes de la médecine chinoise : le principe du yin et du yang, deux mouvements énergétiques complémentaires, et le principe des cinq éléments.

Dans ce système, la maladie est pensée comme le résultat d'un déséquilibre de l'énergie qui circule dans le corps à travers des trajets spécifiques appelés "méridiens ». Le traitement par acupuncture consiste notamment à placer des aiguilles métalliques sur des points précis du corps, sur les méridiens, de manière à agir sur la circulation et l'équilibre général de l'énergie vitale. Plus précisément, la maladie est définie comme un trouble de l'énergie dans le corps (appelée "Qi » ou "souffle »), que ce soit un trouble de la circulation (s'il y a blocage, il y a symptôme, par exemple la douleur « : il faut faire " circuler »), de la production (il faut traiter les organes qui produisent le " $\mathrm{Qi}$ ») ou des fonctions du "Qi " (il faut faire " circuler ", " réchauffer ", "diffuser »). Ainsi une maladie apparaît-elle soit dans les méridiens, c'est-à-dire dans le système circulatoire où évolue le " Qi ", soit dans les organes, dans le système organique qui produit et conserve le "Qi ». Les maladies peuvent être dues à des facteurs d'origine externe ou interne :

- les facteurs externes sont les éléments climatiques (vent, froid, chaleur, humidité, sécheresse) qui peuvent léser les organes ou bloquer la circulation du " $\mathrm{Qi}$ " dans les méridiens ;

- les facteurs internes sont essentiellement les excès des six émotions : la colère qui attaque plus spécifiquement le foie, la tristesse le poumon, la joie le cœur, les soucis/ ruminations qui attaquent la rate, la peur/crainte qui

5. Interroger ici la notion de tradition associée à cette médecine dépasse largement le cadre de cet exposé. Le lecteur pourra notamment se reporter à l'article d'Elisabeth Hsu (2007) ; en ce qui concerne l'histoire de l'acupuncture en France à partir des années 1930 jusqu'à aujourd'hui, voir également Lucia Candelise $(2008,2010)$. 
attaque le rein. Les émotions sont ici considérées comme des causes internes parce qu'elles sont générées par l'individu, chaque individu ne réagissant pas de la même façon à un même stimuli émotionnel, en fonction de son caractère, de son passé, de sa culture.

Contrairement à la médecine scientifique occidentale, qui pense essentiellement la maladie en termes de dysfonctionnements organiques, l'acupuncture aurait plutôt tendance à opposer une vision du corps en tant que dynamique globale. En fait, l'objet du traitement est d'agir sur les défenses naturelles de l'organisme, qu'il cherche à renforcer plutôt qu'à remplacer.

L'observation et l'analyse des corpus filmiques permettent de dégager quelques pistes de recherche que j'aimerais maintenant présenter. Toutefois, je précise que les résultats de cette étude sont plus à prendre comme des indicateurs ethnologiques que comme des données généralisables à l'ensemble des acupuncteurs français. Dans une optique de comparaison, il serait particulièrement intéressant de pouvoir confronter les résultats aux praticiens des autres principales associations d'acupuncture françaises comme par exemple l'Association française d'acupuncture (AFA), cependant quelques tendances propres au milieu enquêté peuvent être mises en évidence dès à présent.

\section{La plainte du malade : un récit guidé aux frontières biographiques}

L'analyse des consultations dites des "premières fois ", pour reprendre les termes des médecins-acupuncteurs, est riche d'enseignements. En effet, dans le cadre du dialogue qui s'instaure entre le médecin et le patient, se mettent en scène aussi bien des savoir-faire professionnels médicaux propres aux cadres nosologiques de l'acupuncture que les habitus sociaux des patients. Ainsi observet-on du côté des "nouveaux " patients que l'expression de leur plainte est marquée par une narration ${ }^{6}$ qui oscille entre une énonciation objectivée des maux à travers une conception bio-organique des symptômes, mettant les parties du corps souffrant au centre de la plainte et, dans le même temps, une volonté de rattacher ce vécu de la maladie à des événements de leur vie sociale. Aussi, peut-on faire

6. À ce propos, voir également Garro et Mattingly (2000). 
l'hypothèse que la manière des patients de traduire leurs maux en termes de réalité organique est largement élaborée et influencée par le contact avec la pratique médicale allopathique dépositaire d'une vision ontologique du corps et de la maladie. L'examen critique des itinéraires thérapeutiques montre effectivement que les patients consultent généralement le médecin-acupuncteur en dernier recours, une fois que les traitements proposés par la médecine classique n'apportent pas ou plus la satisfaction escomptée.

L'analyse montre que les patients interrogés à ce sujet, à l'exception d'un seul, disent tous venir consulter l'acupuncteur d'abord dans une recherche pragmatique de soin, l'objectif visé étant l'amélioration ou la guérison des maux, ce qui contredit l'image stéréotypée parfois véhiculée de patients en quête d'ésotérisme et d'exotisme médical, même si ce profil existe parfois. Cette composante sociologique aura d'ailleurs des répercussions fortes sur la manière dont les médecins choisiront leurs mots pour expliquer le diagnostic chinois et les points d'acupuncture choisis pour traiter les problèmes. Ainsi, lorsque dès les premières séances les maux disparaissent, il n'est pas rare de constater chez les patients, dans ce contexte d'attente extrême, leur recours à un vocabulaire religieux pour qualifier l'effet thérapeutique perçu : "Ça a été un vrai miracle »; "J’y croyais pas, mais, pourtant ça a marché »; "Maintenant, je suis un adepte, un converti de cette médecine!»

Par ailleurs, les patients parlent aux médecins de leurs problèmes en reprenant la terminologie médicale et un langage propres à la médecine occidentale. L'analyse des consultations filmées montre que les catégories biomédicales utilisées par les patients pour exprimer leurs maux sont pour ainsi dire déconstruites progressivement par l'interrogatoire des médecins-acupuncteurs, ceux-ci ayant recours à une grille de lecture propre à l'interprétation énergétique des symptômes. Par exemple, les patients sont plutôt invités à parler non pas de "leur sciatique ", mais à préciser le trajet des sensations de douleur, l'heure du jour et de la nuit de l'apparition ou de la disparition des symptômes, l'amélioration ou l'aggravation de la douleur au chaud ou au froid, la qualité du sommeil et la nature des rêves, autant d'indices importants aux yeux des acupuncteurs pour établir leur diagnostic médical. Les corpus filmiques révèlent d'ailleurs que les médecins étudiés ont recours à ces deux grilles de lecture 
médicale durant leurs consultations, passant aisément d'un registre à l'autre sans pour autant que cette pratique ne paraisse déplacée aux yeux des patients. Bien au contraire, les entretiens mettent en évidence que ce "bilinguisme médical " (Marcovitch 1987 : 30) dont font preuve les médecins-acupuncteurs formés dans le système médical universitaire classique est plutôt perçu comme un gage de compétence, le médecin étant tour à tour capable d'interpréter le résultat d'un bilan d'examen biologique, comme d'interpréter les émotions du patient dans un tableau clinique énergétique, ce qui contribue également positivement à l'établissement d'une relation de confiance entre soignants et soignés.

Dans le même ordre d'idées, l'analyse fine des images permet aussi de constater combien les éléments biographiques des patients structurent leur discours sur la maladie et leur rapport au médecin : parler de ses maux, c'est d'une certaine manière "se raconter ", c'est un moyen de dire qui l'on est et d'exister en tant qu'individu à part entière. La prise en compte réelle ou supposée de ces éléments par l'acupuncteur constitue par conséquent pour le patient un point d'ancrage fort du travail de la relation thérapeutique accompagnant le traitement par aiguilles proprement dit. En d'autres termes, la maladie en tant qu'événement est la plupart du temps déjà présentée au médecin comme socialisée, intégrée dans un " réseau sémantique " (Good 1998 : 344) qui fait sens pour le patient et qui dépasse pour lui le strict cadre de l'expérience corporelle de ses maux. Si les éléments biographiques n'apparaissent pas comme un trop-plein de sens aux yeux du patient, ils sont en revanche souvent perçus comme un trop-plein de signes à interpréter pour le médecin. C'est ici le statut même de la parole qui fait problème, puisque c'est elle qui délivre soit de façon implicite, soit de manière très ouverte, la manière dont les patients s'expliquent leurs maux. Autrement dit, c'est précisément à travers ce discours sur leurs troubles que les patients font part aux médecins de leurs représentations profanes. Aussi, l'intégration de cette signification profane comme élément de diagnostic varie-t-elle selon la position que les acupuncteurs adoptent face à la valeur épistémique de ces indices symptomatologiques et de leur pertinence pour la médecine chinoise. Certains médecins estiment que l'interprétation de ces «indices profanes » relève davantage du travail du psychanalyste que de celui de l'acupuncteur, sans pour autant nier l'intérêt de ce récit biographique et son importance pour 
le patient ; il est alors intégré au tableau clinique au même titre que les autres signes cliniques (examen de la langue, prise du pouls, etc.) pour établir le choix des points à piquer. D'autres médecins, ayant par exemple suivi une formation en psychologie ou dans le domaine des thérapies brèves, accordent une place plus importante à ces éléments, mais sans pour autant centrer leur diagnostic sur ces seules données.

D'une manière générale, j'ai pu observer que lorsque ces éléments de discours semblaient trop éloignés de la demande formulée par le patient de ce qui était perçu comme relevant du champ de l'acupuncture, les médecins avaient tendance à recentrer ce récit et à le guider, à travers leurs questions, sur les liens établis entre le vécu biographique des maux et leurs expressions, sensations et manifestations corporelles. Laction thérapeutique sur le corps devenait alors l'enjeu majeur de la consultation et l'objet concret du soin. Parfois, j'ai pu observer un usage inverse de la relation au corps, non plus envisagé comme finalité du soin, mais d'abord comme support à l'expression verbale des maux. Dans ce cas, le corps du patient opère comme un « objet neutre » où le détour par l'évocation des sensations corporelles permet au médecin d'aborder des sujets plus intimes concernant la vie sociale du patient : problèmes familiaux, relations de couple, sexualité, relations de conflits au travail.

\section{Effets thérapeutiques et ambivalence des interprétations médicales}

L'étude des consultations filmées permet également de mettre en évidence l'évolution du statut de la parole au sein de la consultation. L'examen approfondi des images montre un changement de ton et de nature des propos échangés au fur et à mesure du déroulement de la consultation. J'ai montré ci-dessus combien le temps de l'interrogatoire situait la parole de façon privilégiée du côté du patient et l'écoute du côté du médecin, sa parole étant plutôt tour à tour celle de l'enquêteur, celle qui rassure et qui encourage, qui compatit ou encore celle qui plaisante. Cet échange ritualisé du début de la consultation se déroule autour du bureau qui sépare les protagonistes de l'action dans l'espace, mettant ainsi une distance physique entre eux, fixant d'une certaine manière une posture des discours attendus dans une improvisation réglée des rôles. 
Il est intéressant de constater combien les échanges sont plus détendus et fréquents durant les moments de transition, par exemple lorsque le patient quitte sa chaise pour se déshabiller avant de s'allonger sur la table d'examen. Quand le médecin ne sort pas de la pièce pour laisser le patient se déshabiller seul, il n'est pas rare que les échanges portent alors sur la famille, les enfants ou encore le travail de ce dernier. Cette discussion moins formelle permet au médecin de maintenir un lien avec le patient ; c'est toujours une façon de l'accompagner et d'assurer en quelque sorte le suivi de la relation thérapeutique, mais sur un autre registre que celui de la maladie. Il n'est pas rare que le patient livre alors de précieuses informations sur son état, qui avaient été laissées hors du champ de l'interrogatoire initial. Au moment de l'examen clinique, qui consiste à observer la langue du patient et à prendre directement son pouls sur ses poignets sans l'intermédiaire d'un instrument médical, le médecin est assis près du malade dans un rapport de forte proximité des corps. Lorsque les échanges de paroles ont lieu, après un moment de silence, ils sont brefs, presque murmurés, marquant ainsi une relation entre le médecin et son patient désormais orientée vers une écoute intime du corps. "À-ce-moment-là, je me tais car c'est important, il me visite !" me confiera une patiente au cours d'un entretien. Souvent, c'est précisément ce moment que choisissent les patients pour tenter de demander au médecin ce qu'il a trouvé : "Alors, docteur ? "Généralement, les médecins évitent de livrer tout de suite une réponse au patient, préférant la remettre à plus tard, une fois qu'il est rhabillé, tout en lui faisant sentir que le moment n'est pas le plus opportun : "Je veux bien parler de ça avec vous, mais pas maintenant, je crois que là ce n'est pas tout à fait le moment, voyezvous... » Juste après vient le temps du traitement proprement dit, celui de la puncture. Le médecin est concentré et peu enclin à parler, sauf lorsqu'il demande au patient de changer de posture pour atteindre une partie de son corps à piquer ou lorsqu'il lui demande d'indiquer la zone la plus sensible à la palpation à traiter. Une fois piqué, le patient est laissé seul, allongé une vingtaine de minutes sur la table d'examen, avec les aiguilles. Parfois, pour ne pas qu'ils aient froid, les médecins recouvrent les patients soit avec leurs propres vêtements en été, soit avec une couverture en hiver, dans une gestuelle qui évoque le bordage d'un enfant, geste de maternage auxquels les patients semblent très sensibles. Maintenant, le médecin quitte la pièce en prononçant une 
parole qui vient clore la puncture : "Je vous laisse un moment ! "; "Détendez-vous, je vous laisse vous détendre! "; "Profitez bien de ce moment, à tout à l'heure! » Commencent alors pour les patients et selon leurs propres termes un dialogue intérieur ", un abandon au sommeil ", " un temps de calme, une parenthèse dans la journé. " Une fois la séance de puncture écoulée, le médecin regagne la pièce de consultation et « dépique » le patient. C'est également un moment de transition important où, le patient peut renseigner le médecin sur les sensations éprouvées pendant la séance. C'est aussi le moment que certains médecins choisissent pour faire part de leur point de vue sur les troubles du patient, livrant ainsi leur interprétation énergétique des maux, pendant que ce dernier se rhabille pour venir s'installer au bureau du médecin.

Intervient alors un moment clef de la consultation du point de vue du dialogue qui s'instaure entre les protagonistes, de la construction de la relation thérapeutique et sans doute aussi de son efficacité. En effet, le langage imagé de la médecine chinoise auquel ont recours les acupuncteurs pour expliquer aux patients leurs maux est souvent perçu par ces derniers comme en partie accessible et fait écho en eux d'une manière d'autant plus forte que ce langage médical singulier semble véhiculer des modèles de représentation du corps, de la maladie et du mal proches de leurs représentations profanes. Cette apparente adéquation entre le modèle de représentation du médecin et celui du malade permet alors à ce dernier de mieux saisir l'action du médecin, d'intégrer la logique symbolique et pratique du soin comme un acte thérapeutique dont il maîtrise pour ainsi dire les enjeux. Je citerai en exemple une patiente venue consulter le médecinacupuncteur à la suite d'une rupture amoureuse. Après avoir entendu la plainte et traité la patiente, le médecin lui a expliqué avoir piqué le méridien du cour, ce qui, dans le contexte de la rupture amoureuse, semble parfaitement avoir été adapté à sa demande, le cour étant dans nos sociétés l'organe associé au centre des émotions et à celui des représentations amoureuses.

Cependant, ce langage symbolique de la médecine chinoise utilisé par les acupuncteurs peut également se révéler plus ambivalent et source de malentendus. En effet, une autre patiente venue consulter chez ce même médecin pour des douleurs cervicales semble avoir été déstabilisée par les mots du médecin lui expliquant qu'il traitait 
chez elle entre autres le foie, ce qui ne correspond pas en termes d'énergétique chinoise à l'organe biomédical qu'est le foie, mais plutôt à un sens élargi impliquant des troubles physiques et émotionnels pouvant donner lieu à un déséquilibre général. Ce malentendu a donc obligé le médecin à entrer dans les détails du système de fonctionnement de l'acupuncture en essayant de trouver les mots justes.

Cet épisode est particulièrement intéressant du point de vue de l'étude, car il révèle que les ressources mobilisées par les médecins pour adapter leur langage au niveau sociologique supposé des patients tiennent justement compte des éléments biographiques distillés lors des interrogatoires. En d'autres termes, les médecins semblent adapter leurs discours en fonction de l'idée et de l'image qu'ils peuvent se faire du patient, de sa capacité à comprendre ou à entendre l'interprétation énergétique chinoise de ses maux, courant ainsi le risque d'une possible surinterprétation profane qui se révélerait finalement parfois contraire à l'effet thérapeutique recherché.

\section{Conclusion}

Au stade de cette recherche, il est encore trop tôt pour dire si l'acupuncture permet un modèle de mise en récit des maux très différent de celui de la médecine biomédicale, mais certains éléments de l'enquête permettent au moins d'avancer que le sens profane que les patients attribuent à leurs maux est ici entendu, attitude qui, comme je l'ai montré, débouche sur une certaine forme d'ambivalence. Mais quoi qu'il en soit, cette représentation profane n'est pas perçue par le médecin comme une interprétation erronée ; au pire elle ne suscite de sa part aucune manifestation, ni approbation ni désapprobation, laissant ainsi le patient libre de penser que ces données jouent un rôle dans la prise en charge globale de ses maux.

Les données filmiques produites et proposées à l'analyse critique des enquêtés (médecins et patients) sous la forme de premiers montages m’ont donc été très utiles pour évaluer la manière dont la charge émotionnelle de certaines consultations avait pu influencer mes choix de cadrage au tournage. En effet, lors des séances de restitution des films aux enquêtés, j'ai pu constater combien j'avais 
souvent privilégié le patient au détriment du médecin dans mes choix de réalisation, étant moi-même parfois trop affecté par le discours du patient. J'ai également constaté combien l'expression des émotions par les patients (pleurs, tristesse, joie, rires, etc.) était induite par la grille d'analyse à laquelle les médecins avaient recours. En effet, l'étude de l'acupuncture, faite de correspondances et d'analogies, permet au thérapeute d'aborder tous les champs de la vie du patient (soma, psyché, émotions...), car c'est de tous ces faits qu'il tire les informations propices à la bonne marche de son raisonnement médical.

Ces allers-retours entre le terrain et les données filmiques m’ont donc permis de construire un objet filmique non clos, ouvert sur d'autres solutions, tant cognitives que narratives, tenant mieux compte du point de vue des enquêtés et de leur vécu. La réflexivité qu'offre l'image dans l'enquête ethnologique, méthode déjà expérimentée sur d'autres terrains (Remillet 2009a), a donc joué un rôle majeur dans la connaissance approfondie des relations thérapeutiques observées et s'est avérée précieuse pour limiter les possibles surinterprétations autant que pour valider ou infirmer les hypothèses de recherche et approfondir la connaissance du milieu étudié. Ce corpus de données ethno-filmées a donc été pensé comme la matière première de l'investigation anthropologique, à la fois support de connaissance ethnologique, d'échange avec les enquêtés (médecins et malades) et de communication scientifique, par la réalisation d'un film documentaire ethnologique de synthèse en cours de montage, Rencontre(s) avec les homme(s) (90 min, HD, 2012).

$\mathrm{Si}$, pour les besoins de notre réflexion, nous avons séparé de manière arbitraire le domaine du corps, des émotions et du récit dans la consultation médicale d'acupuncture, l'observation filmée des relations de soin révèle quant à elle leur étroite imbrication, tant dans les comportements que dans les discours à la frontière des normes, des valeurs et des représentations.

D’une manière générale, il nous semble important d'attirer l'attention sur les possibilités heuristiques qu'offre le film pour une anthropologie des relations médecin/malade en acupuncture et, audelà, des pratiques thérapeutiques en situation clinique, domaine de l'anthropologie visuelle encore peu exploré. 


\section{Références}

Aledo-Remillet, Karine et Gilles Remillet, 2004, Malades, médecins et épilepsies. Une approche anthropologique. Paris, l'Harmattan.

Benoit, Jean (dir.), 1996, Soigner au pluriel. Essai sur le pluralisme médical. Paris, Karthala.

Candelise, Lucia, 2008, La médecine chinoise dans la pratique médicale en France et en Italie, de 1930 à nos jours. Représentations, réception, tentatives d'intégration. Thèse de Doctorat, EHESS.

--, 2008, "Construction, acculturation et intégration de l'acupuncture traditionaliste française au XX $\mathrm{XX}^{\mathrm{e}}$ siècle ». Documents pour une histoire des techniques $16: 76-78$.

--, 2010, "Georges Soulié De Morant. Le premier expert français en acupuncture ». Revue de synthèse, Tome 131, 6e série (3) : 373-399.

Crécy,Hélène de, La consultation, 94 min, couleurs, Production,Arthuro Moi. Coproduction, Intrepid Pictures, Distribution DVD Ad Vitam, MK2, 2008.

Desjeux, Dominique (dir.), 2001, La pratique de l'acupuncture en France. Paris, Sorbonne.

Fainzang, Sylvie, 2006a, "L'anthropologie médicale en France ». Dans Francine Saillant et Serge Genest, Anthropologie médicale. Ancrages locaux, défis globaux. Paris, Les Presses de l'Université Laval : 155-173.

_-, 2006b, La relation médecins-malades. Information et mensonge. Paris, PUF.

--, 2001, Médicaments et société. Le patient, le médecin et l'ordonnance. Paris, PUF.

France, Claudine de, 2006, «La profilmie, une forme permanente d'artifice en documentaire ». Dans Claudine de France et Annie Comolli, Travaux en anthropologie filmique. Nanterre, Université Paris X - FRC, "Cinéma et sciences humaines $» 13: 117-142$.

--, 1989 [1982], Cinéma et anthropologie. Paris, Maison des sciences de l'homme.

Garro Linda C. et Cheryl Mattingly, 2000, Narrative and the cultural construction of illness and healing. Los Angeles, University of California Press.

Good, Byron, 1998, Comment faire de l'anthropologie médicale? Paris, Empêcheurs de penser en rond.

Gueronnet, Jane, 1987, Le Geste cinématographique. Collection Cinéma et sciences humaines no. 6, Nanterre, Université Paris X - FRC.

Guilloux, Ronald, 2006, De l'exotisme au politique. La réception de l'acupuncture extrême-orientale dans le système de santé français (XVII - XXe siècles). Thèse de doctorat, Université Lumière-Lyon. 
Hsu, Élisabeth, 2007, «La médecine chinoise traditionnelle en République populaire de Chine. D’une "tradition inventée" à une "modernité alternative” ». Dans Anne Cheng (dir.), 2007, La pensée en Chine aujourd'hui. Paris, Folio-Essais : 214-238.

Marcovitch, Anne, 1987, Les représentations du corps et de la maladie chez les médecins acupuncteurs français et chez leur patients. Paris, CNRS/Inserm. Mino, Jean-Christophe, 2006, « De la médecine clinique à la biomédecine ». Dans Jean-Marc Mouillie, Céline Lefève et Laurent Visier, Médecine et sciences humaines. Paris, Les Belles Lettres.

Petonnet, Colette, 1982, «L'observation flottante. L'exemple d'un cimetière parisien ». L'Homme 21 (4) : 37- 47.

Pouchelle, Marie-Christine, 2003, L'hôpital corps et âme. Essaisd'anthropologie hospitalière. Paris, Seli Arslan.

Remillet, Gilles, 2010, Les aiguilles au fil des maux, réalisé en collaboration avec un patient (n.d) de l'Hérault.

--, 2009a, Ethno-cinématographie du travail ouvrier. Essai d'anthropologie visuelle en milieu industriel. Paris, L'Harmattan.

--, 2009b, «Le travail petit à petit... ». Actes en ligne. Colloque international Jean Rouch Paris : 14-20 novembre 2009. Comité du film ethnographique, session: Une anthropologie partagée. Paris, CNRS.

--, 2009c, «Filmer le travail ouvrier. L'exemple d'une fonderie gardoise ». Dans Corine Eyraud et Guy Lambert (dirs.), Filmer le travail. Films et travail. Cinéma et sciences sociales. Publication de l'Université d'Aix-enProvence : 75-80.

--, 2007, «L'usine, un terrain miné. Récit et analyse d'une insertion en milieu industriel ». Socio-Anthropologie 20 : 135-157.

Sarradon-Eck, Aline, 2008, " Médecin et anthropologue, médecin contre anthropologue : dilemmes éthiques pour ethnographes en situation clinique ». Ethnographiques.org (en ligne), http://www.ethnographiques. org/2008/Sarrondon-Eck.htlm

Schmitz, Olivier, 2006, Les médecines en parallèle. Multiplicité des recours au soin en Occident. Paris, Karthala.

Vega, Anne, 2000, Une ethnologue à l'hôpital. L'ambiguité du quotidien infirmier. Paris, Éditions des archives contemporaines.

Wunenburger, Jean-Jacques, 2006, Imaginaires et rationalité des médecines alternatives. Paris, Les Belles Lettres.

Van der Keuken, Johan, 1998, Aventures d'un regard. Paris, Cahiers du cinéma. 


\section{Documents filmiques de terrain}

-Rencontre(s) avec les homme(s). 2012, HD, coul., 90 min, en cours de montage.

-Les aiguilles au fil des maux. 2010, HDV, coul., $30 \mathrm{~min}$.

\section{Documents filmiques de terrain}

-Rencontre(s) avec les homme(s). 2012, HD, coul., 90 min, en cours de montage.

-Les aiguilles au fil des maux. 2010, HDV, coul., $30 \mathrm{~min}$.

\section{Consultations filmées en milieu hospitalier}

Nîmes

Dr D. D., (6). 2009, HDV, coul., 180 min.

\section{Consultations filmées en milieu libéral}

Montpellier

Dr. F. D., (10). 2009, HDV, coul., 300 min.

Nîmes:

- Dr. JL L., (5). 2009, HDV, coul., 150 min.

- Dr. H. R., (10). 2009, HDV, coul., 300 min.

Perpignan :

- Dr. JP G., (20). 2009, HDV, coul., 600 min- 